\title{
On-line Colour Measurement Using Laser-based Abridged Spectrophotometry
}

\section{Document Version}

Accepted author manuscript

Link to publication record in Manchester Research Explorer

\section{Citation for published version (APA):}

Oulton, D. P., El Sayed, I., \& Porat, I. (1998). On-line Colour Measurement Using Laser-based Abridged Spectrophotometry. Journal of the Textile Institute, 89(1), 63-71.

\section{Published in:}

Journal of the Textile Institute

\section{Citing this paper}

Please note that where the full-text provided on Manchester Research Explorer is the Author Accepted Manuscript or Proof version this may differ from the final Published version. If citing, it is advised that you check and use the publisher's definitive version.

\section{General rights}

Copyright and moral rights for the publications made accessible in the Research Explorer are retained by the authors and/or other copyright owners and it is a condition of accessing publications that users recognise and abide by the legal requirements associated with these rights.

\section{Takedown policy}

If you believe that this document breaches copyright please refer to the University of Manchester's Takedown Procedures [http://man.ac.uk/04Y6Bo] or contact uml.scholarlycommunications@manchester.ac.uk providing relevant details, so we can investigate your claim.

\section{OPEN ACCESS}




\title{
On-Line Colour Measurement Using Laser-Based Abridged Spectrophotometry
}

\author{
David P.Oulton, Ihab El Sayed, and Isaac Porat \\ UMIST Dept of Textiles Manchester UK.
}

\section{ABSTRACT}

Carefully constructed colour relationships and subtle colour choice are central to design inspiration. When the designer's ideas are transferred to production loss of the original colour inspiration is potentially very damaging to the ultimate success of the design. Reliable on-line colour measurement may prove to be an important part of the technology needed to faithfully reproduce original design ideas.

A demonstration Laser photometer, intended to measure colorant concentrations during production, has been constructed at UMIST. It has proved capable of a level of measurement sensitivity and reflectance measurement accuracy, comparable to a reference bench spectrophotometer, at the three wavelengths used.

The intent is to provide an instrument technology, with which the output of a coloration system can be monitored and potentially controlled, on-line. The availability of abundant monochromatic light from lasers, and fibre-optic delivery, makes cheap multi-head measuring systems feasible.

The predicted reflectance values for the laser wavelengths are provided by the match prediction system. The colorimeter compares required with actual reflectance at three wavelengths, and can generate an unambiguous control signal for up to three colorants in the recipe.

Additional laser lines can be added if more than three colorant concentrations need to be controlled.

\section{INTRODUCTION}

The quality and popularity of a textile design depends primarily on the inspiration of the designer. A critical factor in its ultimate success, is the faithful reproduction of the original design idea in production, particularly its subtlety of colour and tone.

In the investigation reported here [1], the authors aimed to produce a measurement system intended for monitoring the concentration of known colorant formulations on-line during production. The objective is to provide improved on-line colour control, avoiding the instrument metamerism and instrument-to-instrument variation inherent in existing three-channel measurement systems. The alternative, of using full spectrum on-line spectro-photometry, has been tried with limited success. The adapted versions of bench spectrophotometers used, do not respond well to an industrial coloration environment.

Laser based Tristimulus Photometry, proposed as a new approach to on-line colour control, uses spectrally differentiated reflectance to generate RGB triplet colour specifications, as an absolute measure of colour. By using a small number of widely separated monochromatic radiation lines, it allows the construction of a potentially cheap rugged on-line abridged spectro-photometer.

The consequences of using just three monochromatic laser-generated wavelengths as the illuminant for reflectance measurement however, require careful analysis. In the following account, the inherent limitations and the practical advantages are discussed. 
The measured RGB reflectance triplets can be compared directly with the expected reflectance values for the known colorants, and metameric effects inherent in conditional matches are avoided.

A set of comparative reference reflectance values for the laser wavelengths is provided by the match prediction system, or generated from an agreed colour standard. The photometer compares desired with actual reflectance at three wavelengths, and can generate an unambiguous control signal for up to three colorants in the recipe.

\section{Three Channel Versus Full-Spectrum Photometry}

Conventional filter-based tristimulus colorimetry is inevitably a compromise. The only truly measurable aspect of colour is the spectrally differentiated power distribution of incident or reflected light. In conventional tri-stimulus colorimeters, the design seeks to simulate the human response. It does this by carefully adjusting the illuminant and colour differentiating filter characteristics to match CIE Standard Observer response characteristics [2].

It is almost inevitable, that the many variables of illuminant output and filter manufacture result in inter-instrument differences [3]. The result is that each individual make, and to some extent each instrument differs slightly from the human Standard Observer response. These differences contribute first to instrument metamerism relative to the Standard Observer. The effect of this is either to incorrectly assign equivalent colour identity to certain metamerically mismatching sample pairs, or assign different identities to visually matching metamers.

The second potential inaccuracy appears as systematic deviation in measured values. This is caused by incorrect channel response characteristics (balance or chromaticity definition), compared to the defined Standard Observer.

By contrast with colorimeters, a full spectrophotometer measures the spectral light flux, and calculation of CIE Standard Observer response is used to establish colour identity in threedimensional XYZ space. In principle all spectrophotometers use identical calculations.

The laser photometer concept arises from the possibility of calculating the CIE colour identity for any mixture of three monochromatic primaries. The calculation is a simple matrix crossdependency, provided primary intensities are expressed in T-Units [4]

The predicted reflectance curve, as the defining colour standard desired (output from recipe prediction)is first generated. An RGB triplet CIE colour identity is then generated from a production dyeing, using lasers. Provided the colorants remain identical, production is potentially an unconditional match to the specified reflectance curve and triplet colour identity is a precise definition of matching quality.

The laser photometer seeks to establish accurately, the measured reflectance at precisely defined wavelengths. The reference standard reflectance values are obtained by interpolation between measured spectrophotometer output values. The photometer uses very narrow band, constant wavelength laser light to re-measure and compare production with target values. The highly monochromatic nature, wavelength stability, and high light intensity of lasers make them an ideal choice for this purpose.

The cost and potential inaccuracy of filters simulating the Standard Observer response is avoided in the proposed measurement method. The central problem of spectro-photometry, that of differentiating responses at precise and adjacent wavelengths is also avoided. Using just three widely separated lines, makes the design of the sensors relatively simple. 
A variety of techniques is available for elimination of stray light effects. They start with narrow band filtering, and at more sophisticated levels can use phase-locked modulated laser beam measurement to differentiate measured light from constant stray light. A full discussion is given by El Sayed [5].

\section{Laser Light}

Laser output is characterized by high intensity, stable wavelength, and ease of collimation [7]. It is thus relatively easy to produce an intense light spot on a surface, even from a meter or more distance. Reflected light can be gathered equally easily, for example via telescope or contact head (see fig 2).

A reasonably large number of Laser-mode light generation systems, provide a wide choice of monochromatic lines for use in a Laser colorimeter. For the instrument described below, the following lines were chosen.

$\begin{array}{lll}\text { Red } & 632.8 \mathrm{Nm} & \text { (Helium-Neon) 10Mw } \\ \text { Green } & 514 \mathrm{Nm} & \text { (Helium-Cadmium) 10Mw } \\ \text { Blue } & 441.6 \mathrm{Nm} & \text { (Argon Ion) 6Mw }\end{array}$

A wide choice of alternatives can be found in the Laser Handbook [7]. The above wavelengths were chosen for widest all-positive coverage, based on position on the spectrum locus.

The intensity of the Lasers chosen (class $3 b^{*}$ ) has been calculated to be sufficient to drive towards 100 measuring heads, given efficient light delivery and collection. In practice, a dozen or so should be relatively easy to achieve with $10 \mathrm{Mw}$ of beam power.

Much cheaper robust solid-state or diode Lasers are available, and could be used in place of the gas Lasers described above [5].

\section{The UMIST Laser Photometer}

A laboratory demonstration Laser photometer has been constructed at UMIST [5]. The demonstration system uses both non-contact (telescope) light collection in 45/0 degree mode, and contact 45/0 mode. See figs 1 and 2.

Considerable work was needed to deliver reliable reflectance measurements using Lasers, as the light output from lasers was found to be significantly unstable in intensity. Fibre optic light delivery also potentially introduces unwanted intensity variation.

El Sayed has shown [5] that all three lasers exhibit light output variation, in the form of long term intensity drift and short period scintillation. Cyclic medium period variation was also encountered. The cyclic variation of the green Laser was found to have a periodicity of about 15 minutes and an amplitude of about $+/-5$ percent.

Short term variation, periodicity 0.1 to 3 seconds peak-to-peak, with an amplitude of $+/-2-3$ percent necessitated averaging up to 250 readings for consistency. Overall measurement frequency achieved was about 650 measurements/Sec for single readings, reducing to 17/Sec, when averaging 250 readings. Better electronics and more powerful computing could raise both rates substantially. 


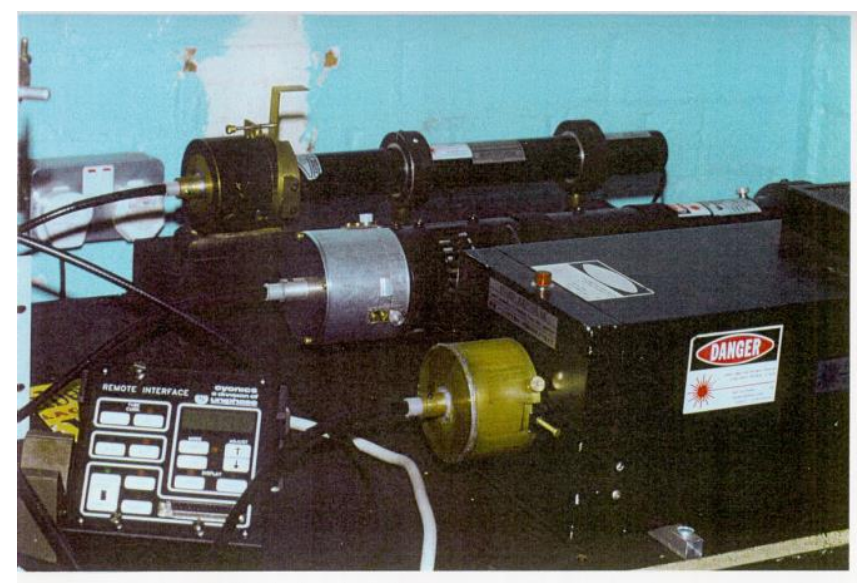

Figure 1. The lasers, electronics, and fibre optics

Figure 3.6. Three lasers with filter housing and optic fibres

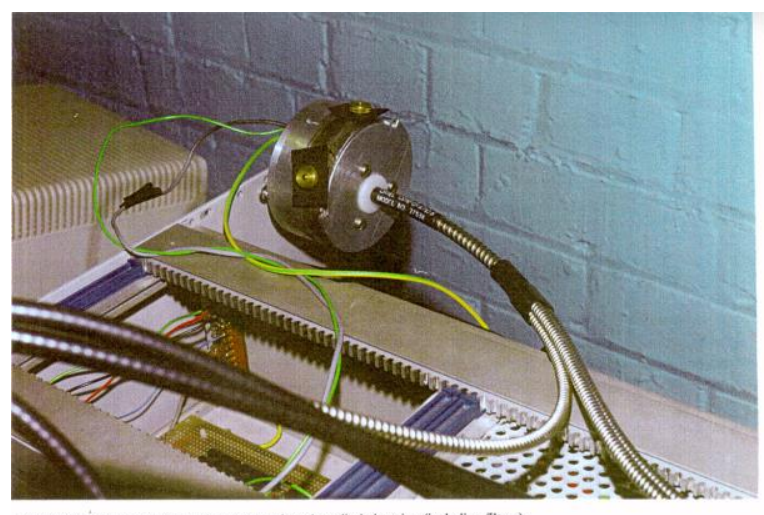

Figure 2. Photo-diode sensor filters and fibre optic light transfer

The object to be measured is illuminated sequentially with the three Lasers, interposing a white reference before each measurement. A photo-diode, A/D converter and electronics, which amplify the signal and filter noise, deliver sensor readings to the computer. A set of three alternative narrow band interference filters, giving 100/1 stray light rejection outside a $10 \mathrm{Nm}$ bandwidth, are place sequentially by a filter wheel in the photo-diode light path.

The Laser photometer is shown to be capable of a level of measurement sensitivity, and reflectance measurement accuracy comparable to the reference Spectraflash SF500, at the three wavelengths used. Individual reading-to-reading variation was estimated at $+/-3$ percent of indicated value. 


\section{RESULTS}

A programme of test measurements, using the BCIRA standard colour tiles, was set up in order to compare standard spectrophotometer reflectance measurement with Laser reflectance values. The narrow bandwidth of laser light required the precise reference reflectance values to be interpolated. Some steeply changing reflectance curves have a slope exceeding 1 percent/Nm. Comparisons were made [5] of full spectral CIE colour identity and RGB triplet CIE colour identity, to determine the effects on colour appearance of using an 'illuminant' with just three wavelengths present. When calculating CIE RGB triplet colour identity, D65 neutral colour balance was used. Strong metameric colour shifts are shown with some of the standard tiles. The calculated CIE RGB triplet colour identities are significantly different from the full spectral Colour identities.

For each of the 12 reference tiles used, reflectance was measured with UV and specular reflectance excluded, using a Spectraflash SF500. The same tiles were then re-measured using the Laser Colorimeter.

Conversion of reflectance triplets to CIE co-ordinate colour identities, was carried out by the matrix method given by Sproson [6]. Precise calculation is achieved, based on the chromaticities of the primaries and the white-point, provided light intensity is expressed in T-Units [4].

For the chosen wavelengths, and a D65 white-point the matrix required is given in the following equation

$$
\left|\begin{array}{l}
\mathrm{X} \\
\mathrm{Y} \\
\mathrm{Z}
\end{array}\right|=\left|\begin{array}{lll}
0.7346 & 0.02831 & 0.1876 \\
0.2975 & 0.6887 & 0.01376 \\
0.000084 & 0.1409 & 0.9481
\end{array}\right| *\left|\begin{array}{l}
\mathrm{R} \\
\mathrm{G} \\
\mathrm{B}
\end{array}\right|
$$

During construction the sensor response of the colorimeter was checked at each of the three wavelengths for linearity of response with CIE Y, and scaled by reference to a standard white tile. The same white tile was used to standardize response during measurement. Linearization and balancing for D65 delivers T-Unit sensor response. A discussion of T-Unit measurement is given by Oulton and Porat in [8].

Results of the reflectance measurements are given in Table 1, and correlation between Spectraflash measurements and Laser colorimeter results is shown in Figures 3 - 5. 


\begin{tabular}{|l|c|c|c|c|c|c|}
\hline \multicolumn{7}{|c|}{ Spectro Versus Laser Reflectance Values } \\
\hline & $441.6 \mathrm{~nm}$ & \multicolumn{2}{c|}{$514 \mathrm{~nm}$} & \multicolumn{2}{c|}{$632.8 \mathrm{~nm}$} \\
\hline & SF 500 & Laser & SF500 & Laser & SF500 & Laser \\
\hline Light Grey & 55.1 & 51.8 & 59.1 & 60.7 & 58.1 & 54.5 \\
\hline Medium. Grey & 25.5 & 23.4 & 26.0 & 25.8 & 23.4 & 22.0 \\
\hline Dark Grey & 5.71 & 5.89 & 5.03 & 4.96 & 4.97 & 5.03 \\
\hline Maroon & 1.44 & 2.02 & 0.830 & 0.918 & 8.59 & 8.14 \\
\hline Pink & 28.3 & 25.0 & 36.6 & 36.5 & 73.3 & 70.2 \\
\hline Brown & 3.97 & 4.06 & 7.22 & 6.92 & 41.9 & 39.5 \\
\hline Yellow & 6.40 & 5.95 & 46.8 & 47.5 & 73.9 & 69.0 \\
\hline Dark Green & 2.36 & 2.53 & 6.56 & 6.52 & 4.65 & 4.35 \\
\hline Light Green & 18.8 & 17.4 & 35.1 & 35.8 & 24.0 & 22.4 \\
\hline Green-Blue & 8.50 & 7.95 & 10.9 & 10.8 & 3.48 & 3.51 \\
\hline Medium.Blue & 23.5 & 22.4 & 15.0 & 14.7 & 8.12 & 7.59 \\
\hline Dark.Blue & 7.79 & 7.19 & 1.25 & 1.18 & 0.779 & 1.15 \\
\hline \multicolumn{7}{|c|}{8} \\
\hline White ref & 80.4 & 80.4 & 84 & 84 & 78.6 & 78.6 \\
\hline
\end{tabular}

Table 1. Comparison of SF500 interpolated reflectance and values measured using the Laser photometer.

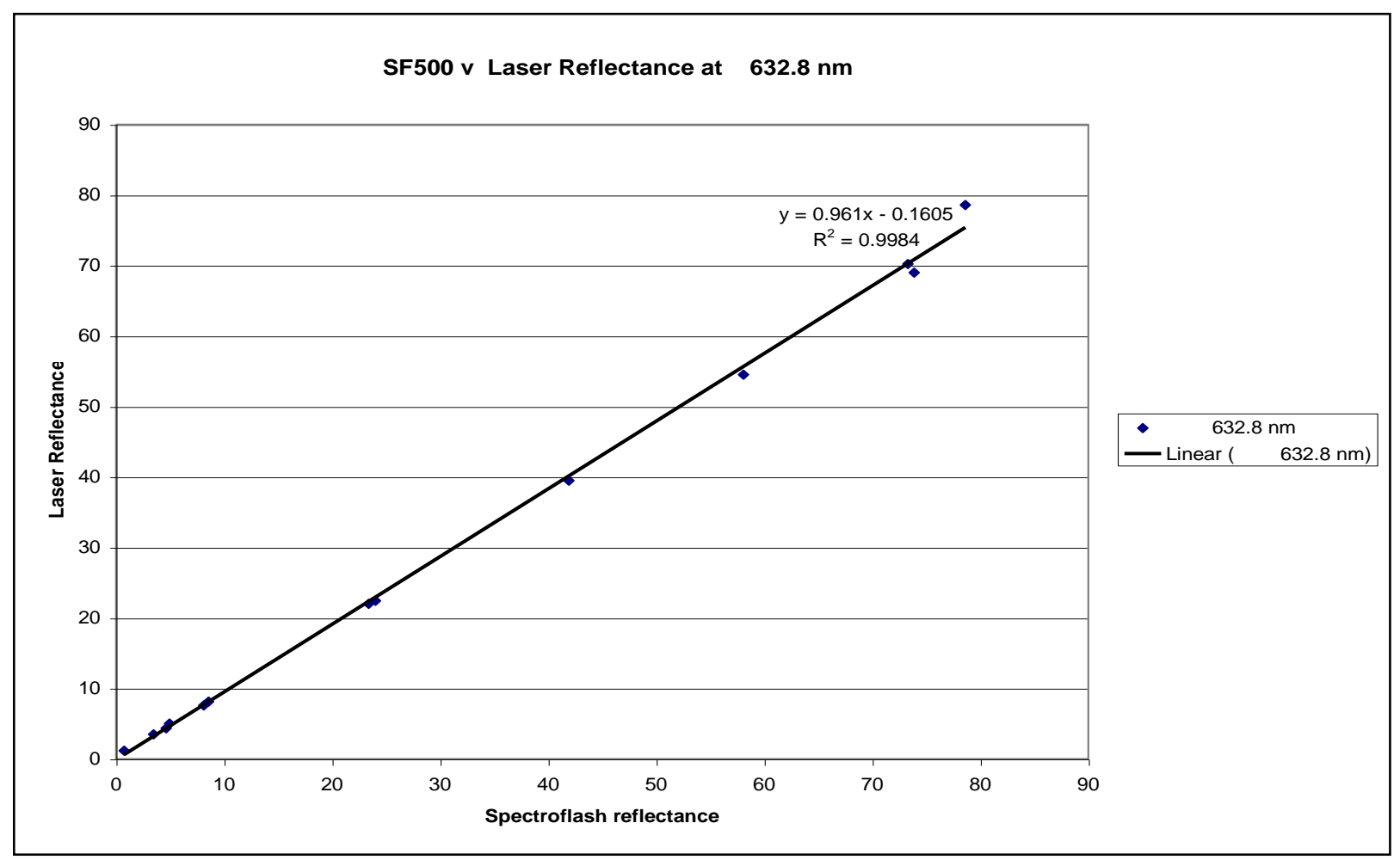

Figure 3.Laser Reflectance as a function of interpolated SF500 reflectance values at $632.8 \mathrm{~nm}$ 


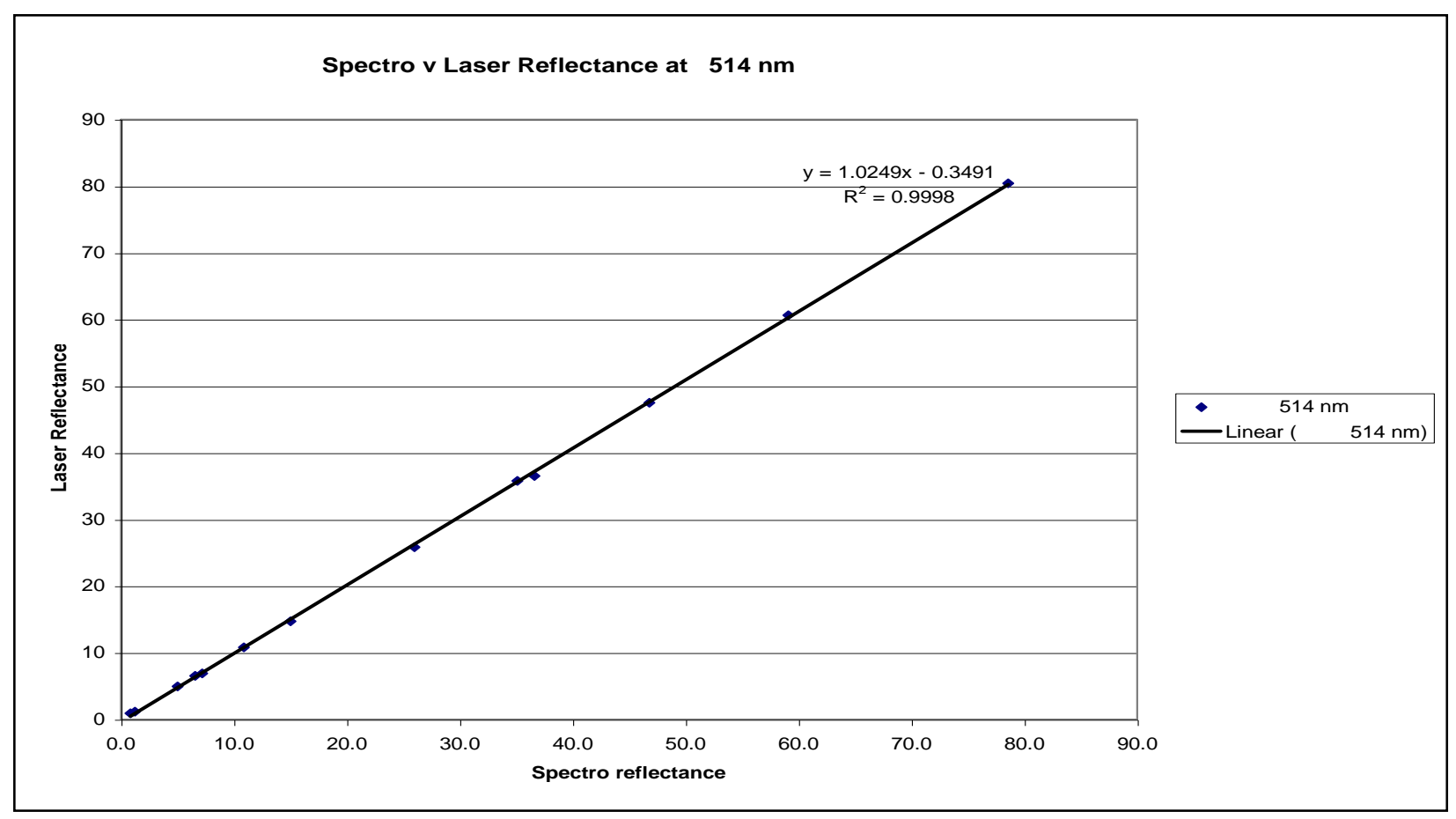

Figure 4. Laser reflectance as a function of interpolated SF500 reflectance values at $514 \mathrm{~nm}$

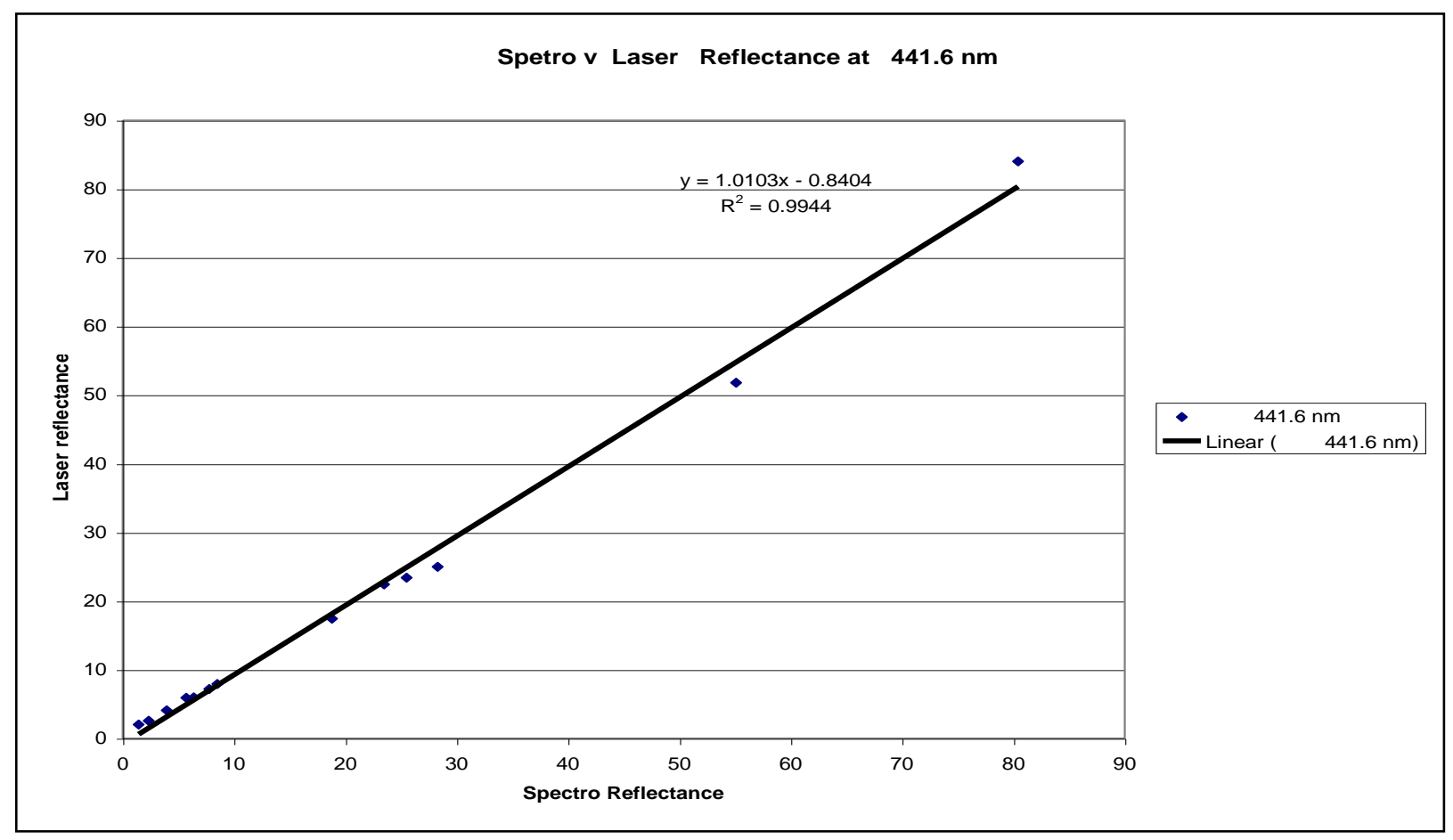

Figure 5.Laser reflectance as a function of interpolated SF500 reflectance values at $441.6 \mathrm{~nm}$ 


\section{Chromaticity And Lightness Differences}

Based on the RGB triplet reflectance values, CIE XYZ colour identities can be calculated. The values are given in Table 2 . below, and analyzed as chromaticity and lightness differences in Table 3.

\begin{tabular}{|c|c|c|c|c|c|c|}
\hline & \multicolumn{6}{|c|}{$\begin{array}{l}\text { Calculated CIE } 2 \text { Degree Obs XYZ Values } \\
\text { Based on Reflectance at three Wavelengths }\end{array}$} \\
\hline \multirow[b]{3}{*}{ White ref } & \multicolumn{3}{|c|}{ Spectro } & \multicolumn{3}{|c|}{ Laser } \\
\hline & $\bar{X}$ & $\mathrm{Y}$ & $\bar{Z}$ & $\bar{X}$ & $\mathrm{Y}$ & $\bar{Z}$ \\
\hline & 75.1 & 82.3 & 88.0 & 75.1 & 82.3 & 88.0 \\
\hline Light Grey & 54.7 & 58.7 & 60.6 & 53.2 & 58.9 & 57.6 \\
\hline Medium. Grey & 22.7 & 25.2 & 27.8 & 22.0 & 24.8 & 25.8 \\
\hline Dark Grey & 4.86 & 5.02 & 6.12 & 5.10 & 5.04 & 6.29 \\
\hline Maroon & 6.61 & 3.15 & 1.49 & 6.65 & 3.26 & 2.13 \\
\hline Pink & 60.2 & 47.4 & 32.0 & 59.4 & 47.4 & 28.8 \\
\hline Brown & 31.7 & 17.5 & 4.78 & 31.2 & 17.4 & 4.82 \\
\hline Yellow & 56.8 & 54.3 & 12.7 & 55.2 & 54.2 & 12.0 \\
\hline Dark Green & 4.04 & 5.93 & 3.16 & 4.14 & 5.89 & 3.29 \\
\hline Light Green & 22.1 & 31.6 & 22.7 & 21.4 & 31.5 & 21.4 \\
\hline Green-Blue & 4.46 & 8.63 & 9.58 & 4.47 & 8.51 & 9.06 \\
\hline Medium.Blue & 10.8 & 13.1 & 24.3 & 10.5 & 12.6 & 23.3 \\
\hline Dark.Blue & 2.07 & 1.20 & 7.55 & 2.26 & 1.26 & 7.03 \\
\hline
\end{tabular}

Table 2. Comparison of Triplet CIE co-ordinates, derived from SF500 data and Laser measurement data

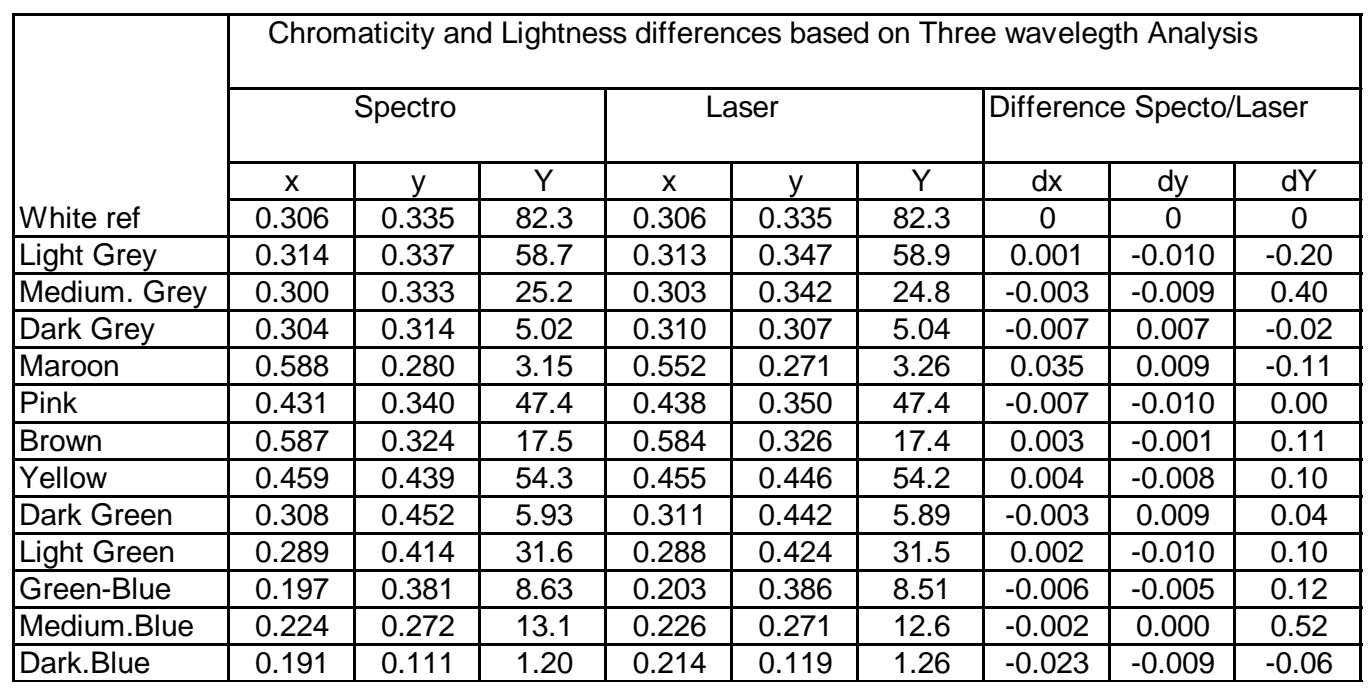

Table 3. Comparison of derived x,y chromaticity and Y for SF500 and Laser Colorimeter 
Although the demonstration instrument is a long way from what might be used on the 'shop floor' it shows promising performance.

Some departure from linear response is shown by the Laser colorimeter at reflectance values below 2 percent and above 80 percent.

\section{Conclusions}

1. The loss of design inspiration due to inaccurate colour reproduction in the final product is potentially very damaging. It was the aim of the authors in developing the Laser Colorimeter, to improve the control of production colour.

2. The system developed is in essence an abridged spectrophotometer. As such it produces a purely physical measurement of the colour under analysis. Because it uses just three wavelengths for comparison, it is limited to monitoring the quality of unconditional or nonmetameric matches.

3. The high light intensity, inherently stable wavelength, very narrow bandwidth, and ease of collimation of Laser light, makes accurate determination of mono-chromatic reflectance values relatively easy.

4. Without the benefit of sophisticated optical engineering, or complicated monochromators, adequate reflectance values have been determined at three very precise wavelengths.

5. It is suggested that the principles described can be applied to both reflectance and transmission measurement. Contact and remote sensing measurement have both been demonstrated using the UMIST Laser Photometer Demonstration system.

6. The advent of suitable solid-state and diode lasers makes a cheap compact version possible, suitable for building into colorant delivery systems, such as hard-copy colour printers.

7. The powerful gas lasers used have been calculated to produce enough light to drive many individual measuring heads.

\section{Bibliography}

1. Ihab M. Elsayed PhD project "Feedback Control of Dyeing" $1993-1995$

2. F.W.Billmeyer and M.Saltzman "The Principles of Color Technology" $2^{\text {nd }}$ Ed, Publ. By John Wiley USA, 1981

3. R.S. Hunter "Photo electric Tristimulus Colorimetry with three filters" J.Opt.Soc.Am 32 508538,1942

4. W.D.Wright "The Measurement of Colour" $4^{\text {th }}$ Ed. Publ. By Adam Hilger Ltd., London 1969

5. Ihab M. Elsayed "Feedback control of Dyeing" PhD Thesis UMIST England 1995.

6. W.N.Sproson "Colour Science in Television and Display systems" Publ. By Adam Hilger 1983.

7. Arecchi and Schultz-Dubois (editors) "Laser Handbook" vol 1 part A Basic theory and Laser Physics, Part E Physical applications.

8. D.P.Oulton and I.Porat "Control of colour by measurement and feedback". J.Text.Inst Vol 83 No.3 1992 p 454. 